\title{
A Lithium-ion Battery RUL Prediction Method Considering the Capacity Regeneration Phenomenon
}

\author{
Xiaoqiong Pang ${ }^{1, * \mathbb{D}}$, Rui Huang ${ }^{1}$, Jie Wen ${ }^{2}$, Yuanhao Shi ${ }^{2}{ }^{\mathbb{D}}$, Jianfang Jia ${ }^{2}$ and Jianchao Zeng ${ }^{1}$ \\ 1 School of Data Science and Technology, North University of China, No.3, XueYuan Road, \\ JianCaoPing District, Taiyuan 030051, China; 18834169601@163.com (R.H.); zengjianchao@263.net (J.Z.) \\ 2 School of Electrical and Control Engineering, North University of China, No.3, XueYuan Road, \\ JianCaoPing District, Taiyuan 030051, China; wenjie015@gmail.com (J.W.); yhshi@nuc.edu.cn (Y.S.); \\ jiajianfang@nuc.edu.cn (J.J.) \\ * Correspondence: xqpang@nuc.edu.cn; Tel.: +86-0351-3923623
}

Received: 11 May 2019; Accepted: 8 June 2019; Published: 12 June 2019

\begin{abstract}
Prediction of Remaining Useful Life (RUL) of lithium-ion batteries plays a significant role in battery health management. Battery capacity is often chosen as the Health Indicator (HI) in research on lithium-ion battery RUL prediction. In the rest time of batteries, capacity will produce a certain degree of regeneration phenomenon, which exists in the use of each battery. Therefore, considering the capacity regeneration phenomenon in RUL prediction of lithium-ion batteries is helpful to improve the prediction performance of the model. In this paper, a novel method fusing the wavelet decomposition technology (WDT) and the Nonlinear Auto Regressive neural network (NARNN) model for predicting the RUL of a lithium-ion battery is proposed. Firstly, the multi-scale WDT is used to separate the global degradation and local regeneration of a battery capacity series. Then, the RUL prediction framework based on the NARNN model is constructed for the extracted global degradation and local regeneration. Finally, the two parts of the prediction results are combined to obtain the final RUL prediction result. Experiments show that the proposed method can not only effectively capture the capacity regeneration phenomenon, but also has high prediction accuracy and is less affected by different prediction starting points.
\end{abstract}

Keywords: lithium-ion battery; remaining useful life; regeneration phenomenon; wavelet decomposition; NAR neural network

\section{Introduction}

Lithium-ion batteries are considered to be the best energy storage devices for many applications because of their light weight, high energy density, and long life [1-3]. From tiny Bluetooth headsets, cameras, mobile phones, and laptops to hybrid electric cars and aerospace power systems, batteries are very important and critical components. At the same time, battery failures can lead to performance degradation of the power system, or even directly lead to the failure of the task, and increase costs. Improper management of lithium-ion batteries in electric vehicles may cause fire or even explosions [4], and power system malfunction is the main cause of mission failure in the aerospace field [5-7]. Therefore, it is significant to study the prediction of RUL of lithium-ion batteries in practical applications.

In the field of lithium-ion battery RUL prediction, the gradually degraded battery capacity is often used as an effective health indicator in order to track the attenuated process of the battery. In general, a lithium-ion battery is deemed to fail when its capacity fades by $20-30 \%$ of the rated value [8]. In some online applications of lithium-ion battery health prognostics, impedance, voltage, and current are often used as HIs to reflect the battery degradation $[9,10]$. However, the RUL prediction using capacity as the $\mathrm{HI}$ is generally more accurate and effective than other HIs as the change in battery 
capacity directly reveals the health states of batteries [8]. Therefore, battery capacity as the HI is widely used in the RUL prediction of lithium-ion batteries. Additionally, the procedure of battery degradation is not monotonous, the working process of the battery consists of charging, discharging, and the rest stage. Overall, the capacity of a lithium-ion battery shows a degradation trend during usage because of the side reactions that occur between the electrodes and electrolyte of the battery. However, when the battery rests during charge/discharge profiles, the residual reaction products have a chance to dissipate, thus increasing the available capacity for the next cycle. This phenomenon, which emerges during the use of lithium-ion batteries, is called regeneration. The regeneration phenomenon of lithium-ion batteries can alter the trend of the capacity prediction curve, thus affecting the performance of prognostic models [11,12]. Therefore, it is important to consider the capacity regeneration phenomenon in RUL prediction of lithium-ion batteries. Furthermore, the capacity time series should be considered as a hybrid signal of multi-scale components, where global degradation and local regeneration are signals of different scales. It is necessary to decouple the correlative components from the original capacity time series in RUL prediction for extracting the most useful information [13].

In recent years, the RUL prediction methods for lithium-ion batteries can be divided into two types: model-based and data-driven [7]. The data-driven prediction method has become a current research hotspot because it does not need to analyze the complex internal mechanism and electrochemical reaction process of batteries, only relies on the existing historical monitoring data, and avoids the defects of a complex modeling process and large interference caused by environmental factors. Data-driven methods [8,13-21] for health prediction of lithium-ion batteries usually use machine learning models (support vector machines, logistic regression, and neural networks, etc.) to establish the relationship between monitoring data and system health, so as to track the battery degradation and estimate the RUL of batteries. Most of above methods [14-20] only focus on the global degradation trend of batteries and ignore the regeneration phenomenon in battery rest time. In [21], Deng et al. propose an improved empirical model based on that of Saha et al. [12], where they relax the fixed Coulombic efficiency and estimate it with measured data; the dual EKF estimation is then employed to deal with the coupled problem of parameter and state capacity. However, the experimental results show that the sudden rise in capacity during regeneration is not predicted obviously. In [13], He et al. utilize a wavelet decomposition and Gaussian regression combined method to capture the regeneration phenomenon in battery health prognostics, but the performance is not ideal for capturing the regeneration part. In [8], Yu proposes a method that combines empirical mode decomposition (EMD), logistic regression, and Gaussian regression with consideration of the regeneration phenomenon, where an adaptive moving window is added to the regression process to capture the regeneration of batteries by constantly changing the size of the window. Compared with reference [13], this method performs better in capturing the regeneration section. However, the RUL prediction accuracy of such regression methods $[8,13]$ is greatly affected by different prediction starting points. Therefore, it is still a challenging task to design an appropriate RUL prediction method for lithium-ion batteries which can capture the capacity regeneration phenomenon well and acquire a good prediction performance.

In addition, the degradation process of batteries is a complex, dynamic, and nonlinear electrochemical process [22]; in the use of a battery, the capacity and service life of batteries show an irreversible trend of gradual decline with time. Moreover, the degradation of capacity accelerates in the later cycle life, showing a nonlinear characteristic. Therefore, battery capacity degradation data is a kind of nonlinear time series data based on monitoring. In order to predict RUL better, it is necessary to establish an appropriate model that can effectively deal with capacity degradation data with the characteristics of nonlinear time series. With the rise of neural networks, many neural network based methods have been applied in the field of health management and life prediction [18,23-31]. Among them, the NARNN $[25,26,29,30]$ is a dynamic recurrent neural network with time series prediction capability, which can effectively simulate nonlinear processes and deal with stationary and non-stationary time series. Therefore, this paper chooses NARNN to establish the RUL prediction model of lithium-ion batteries. 
Driven by the desire to capture capacity regeneration effectively and improve prediction accuracy, a novel WDT-NARNN method for lithium-ion battery RUL prediction is proposed based on a combination of WDT and NARNN. The WDT with multi-resolution characteristics is used to decompose the capacity time series in multi-scale to get both the global degradation trend and the local regeneration. The NARNN is utilized to recursively predict global degradation and local regeneration. Finally, the NARs are integrated to achieve RUL prediction of lithium-ion batteries. Therefore, the main contributions and innovations of this paper include the following: (1) The global degradation and local regeneration in battery capacity time series can be separated effectively by WDT, which will be helpful to improve the prediction performance of the prediction model; (2) A combined model based on WDT and NARNN is established to model the local and global tendency of the battery capacity changes, which enables the prediction model to capture the actual capacity decay tendency of batteries effectively.

\section{Related Algorithms}

\subsection{Wavelet Decomposition}

Wavelet decomposition technology is a powerful tool to analyze non-linear and non-stationary time series, which is widely used in various fields of engineering [13,32-35]. The global degradation and local regeneration of battery capacity can be decomposed by using wavelet decomposition technology, and the two parts can be processed respectively to achieve accurate prediction.

Wavelet analysis utilizes the wavelet function $\psi(\omega)$ and scaling function $\varphi(\omega)$ to perform the multiresolution analysis decomposition and reconstruction of the signal. Following the idea used in deriving the Meyer's wavelet, Gilles [32] defines the empirical scaling function as

$$
\varphi_{i}(\omega)= \begin{cases}1, & \text { if }|\omega| \leq(1-\xi) \omega_{i} \\ \cos \left(\frac{\pi \rho\left(\xi, \omega_{i}\right)}{2}\right), & \text { if }(1-\xi) \omega_{i} \leq|\omega| \leq(1+\xi) \omega_{i} \\ 0, & \text { otherwise }\end{cases}
$$

and the empirical wavelet function as

$$
\psi_{i}(\omega)= \begin{cases}1, & \text { if }(1+\xi) \omega_{i} \leq|\omega| \leq(1-\xi) \omega_{i+1} \\ \cos \left(\frac{\pi \rho\left(\xi, \omega_{i+1}\right)}{2}\right), & \text { if }(1-\xi) \omega_{i+1} \leq|\omega| \leq(1+\xi) \omega_{i+1} \\ \sin \left(\frac{\pi \rho\left(\xi, \omega_{i}\right)}{2}\right), & \text { if }(1-\xi) \omega_{i} \leq|\omega| \leq(1+\xi) \omega_{i} \\ 0, & \text { otherwise }\end{cases}
$$

where $\rho(x)$ is any arbitrary function with values in the range $[0,1]$ with the following properties:

$$
\rho(x)= \begin{cases}0, & \text { if } x \leq 0 \\ \text { and } \rho(x)+\rho(1-x) & \forall x \in[0,1] \\ 1, & \text { if } x \geq 1\end{cases}
$$

Then, the inherent mechanism of empirical wavelet transform is based on the formation of adaptive wavelet based filters. In the wavelet analysis, the Mallat algorithm is used to decompose the signals and obtain trend information (low frequency) and regeneration information (high frequency). The decomposition equations are expressed as

$$
\begin{aligned}
& A_{j+1, k}=\sum_{m} h(m-2 k) A_{j, m} \\
& D_{j+1, k}=\sum_{m} g(m-2 k) A_{j, m}
\end{aligned}
$$


where $j$ is the decomposition scale, $k$ and $m$ are translation variables, $h$ is the low-pass filter, and $g$ is the high-pass filter.

The decomposed data is reconstructed by reconstruction algorithm to restore it to the original spatial scale. The reconstruction equation is described as

$$
A_{j+1, k}=\sum_{m} h^{*}(m-2 k) A_{j, m}+\sum_{m} g^{*}(m-2 k) A_{j, m}
$$

where $h^{*}$ and $g^{*}$ are the inverse functions of low-pass and high-pass filters respectively.

\subsection{NAR Neural Network}

NARNN is a kind of dynamic recurrent neural network with time series prediction ability. It forms a discrete nonlinear autoregressive system with endogenous input [26,29], and the mathematical representation of NARNN can be defined as follows:

$$
y(t)=f(y(t-1), y(t-2), \ldots, y(t-d))
$$

where $y(t)$ is the current output, $y(t-1), y(t-2), \ldots, y(t-d)$ are the historical outputs, $d$ is the delay of the network.

NARNN is composed of an output layer, hidden layer, and feedback layer. The function of the feedback layer is to store the previous outputs, which can be regarded as a kind of 'memory' operator. The network structure of NARNN is shown in Figure 1.

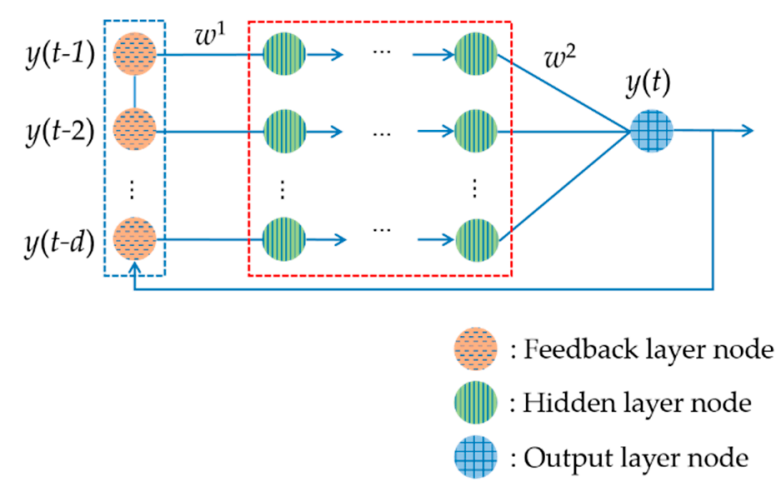

Figure 1. The structure of the Nonlinear Auto Regressive (NAR) neural network, where the $w^{1}$ is the connection matrix between input layer and hidden layer, $w^{2}$ is the connection matrix between hidden layer and output layer.

\section{WDT-NARNN Prediction Method}

\subsection{Experiment Data Analysis}

In this article, the experimental battery data used are derived from the data repository of NASA Ames Prognostics Center of Excellence (PCoE) [36]. Four typical types of 18,650 sized rechargeable batteries (\#5, \#6, \#7 and \#18) were used to illustrate the performance of our proposed approach for RUL prediction of lithium-ion batteries. Figure 2 shows the true capacity degradation curves of the four batteries. It can be seen that there is a clear descending trend, namely, the global degradation, and several capacity regenerations (black circles). Obviously, the existence of these capacity regeneration phenomena changes the normal degradation trend of the batteries. 


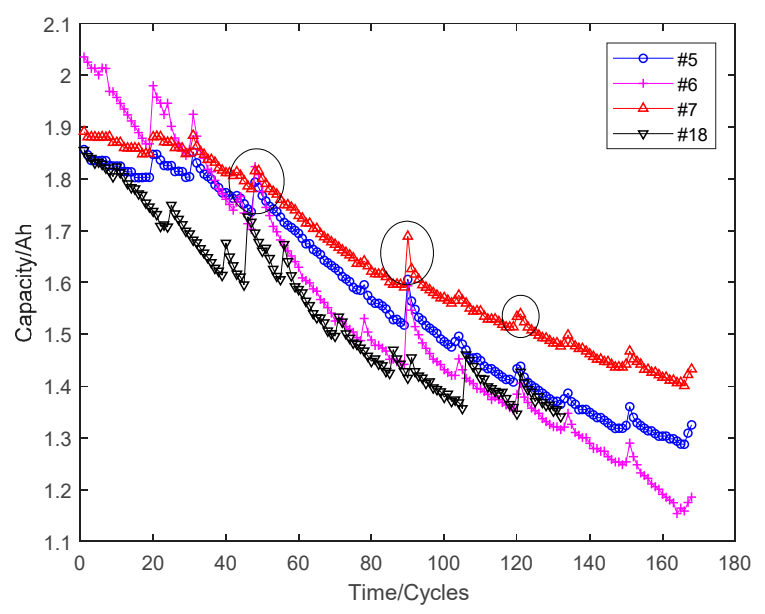

Figure 2. The capacity degradation curves of four batteries (\#5, \#6, \#7, and \#18).

A key step of the proposed model in this paper is to use WDT to decompose the capacity time series at multi-scale to obtain the global degradation trend and local regeneration. Take battery \#5 as an example, the results of 6-layer decomposition by using the 'dmey' wavelet is shown in Figure 3. The curve ' $a 6$ ' in Figure 3(a6) is the low frequency signal, which reveals the normal degradation tendency of the battery capacity. Curve ' $\mathrm{C}$ ' in Figure 3(a6) is the real capacity degradation curve. d1-d6 are the high frequency signals, and the peak value of the fluctuations corresponds to the captured local regenerations. It can be seen that wavelet decomposition can get more degradation information of battery capacity at different scales, and taking these parts into account will be beneficial to improve the prediction accuracy of the RUL prediction model for lithium-ion batteries.

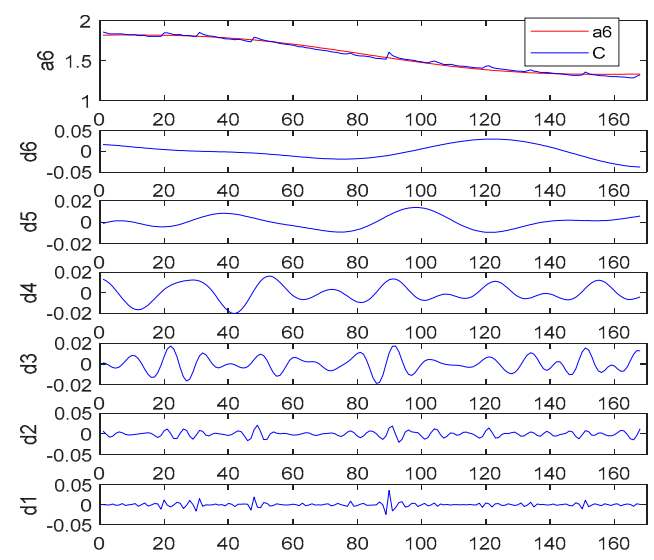

Figure 3. The wavelet decomposition of capacity for battery \#5.

\subsection{WDT- NARNN Modeling Process}

As can be seen from the above decomposition results, the global degradation trend and local regenerations of capacity are quite different in frequency characteristics and shapes. If the prediction is performed directly, it is difficult to capture both features at the same time. Therefore, we proposed a multi-scale decomposition and fusion prediction method WDT-NARNN for the RUL prediction of lithium-ion batteries. The major procedures of the proposed WDT-NARNN modelling method is shown in Figure 4, which is mainly divided into the following steps: 


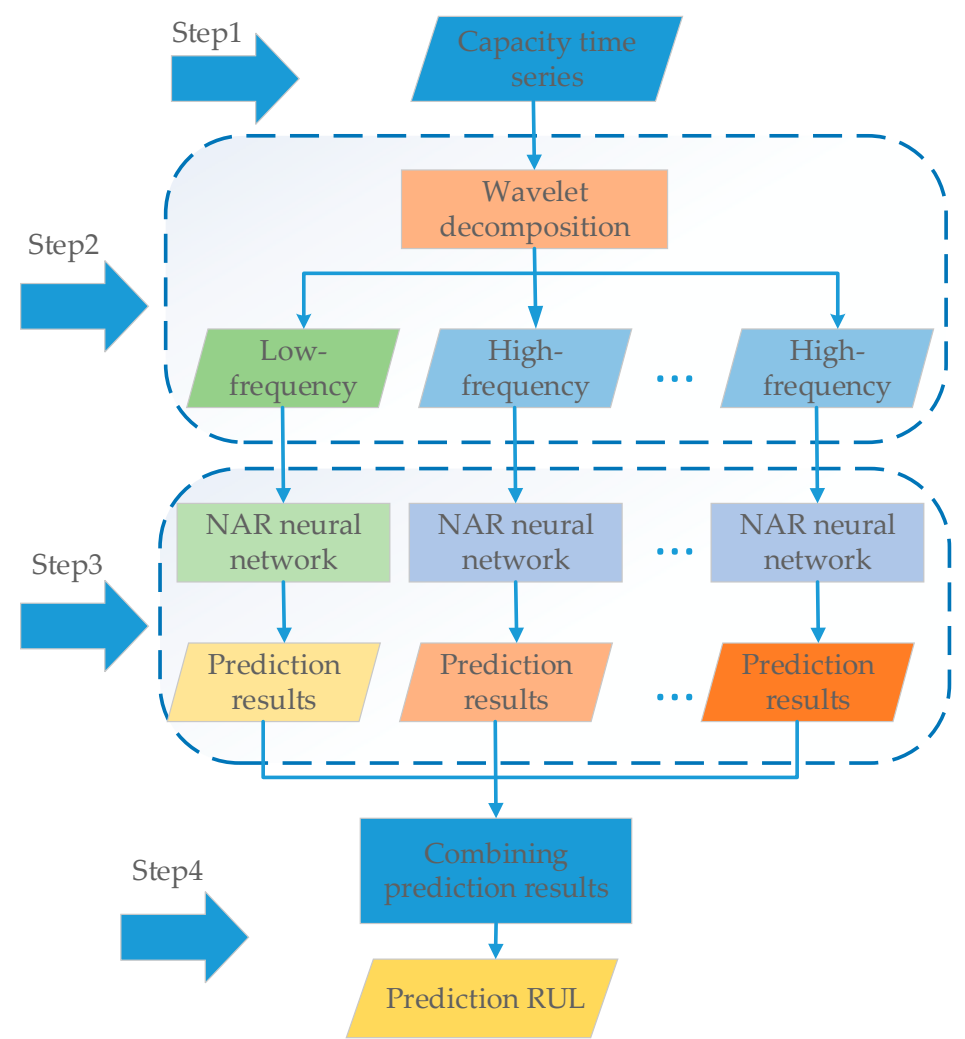

Figure 4. Procedures of lithium-ion battery Remaining Useful Life (RUL) prediction based on the proposed method.

(1) Extract the raw battery capacity series $\{\operatorname{Cap}(i), i\}_{i=1}^{N}, N$ means the total cycle number of each battery.

(2) Decomposition of capacity time series. Firstly, wavelet function $\psi(t)$ and the decomposition level $l$ are initialized. Based on that, the raw capacity time series are decomposed into $l$ levels. At the same time, several high frequency signals and one low frequency signal are obtained by low and high pass filters. The low frequency signal reflects the global trend of capacity degradation and high frequency signals reflect local fluctuations of capacity regeneration.

(3) Constructing prediction models for low-frequency and high-frequency parts respectively. The prediction starting point is set to $T$. The low- and high-frequency parts after wavelet decomposition $\left\{W D T \_C a p(i), i\right\}_{i=1}^{T-1}$ are used as the training samples respectively. Then, NAR neural networks for each decomposition component are designed and trained. Next, the trained NAR models are used to predict the later time series data of each decomposed component.

(4) Combining prediction results. The final capacity prediction result is obtained by combining and reconstructing the prediction results of all the NAR models. Then, according to the relationship between the capacity and cycle numbers, the final RUL value is calculated by the following equation:

$$
N_{R U L}=N_{E O L}-N_{E C L}
$$

where $N_{R U L}$ is the remaining useful life. $N_{E O L}$ is the total charge and discharge cycles when the actual battery capacity degrades to the threshold. $N_{E C L}$ is the charge and discharge cycles at the current time of the battery.

For clarity, the integrated method with WDT and NARNN can be summarized in Algorithm 1. 


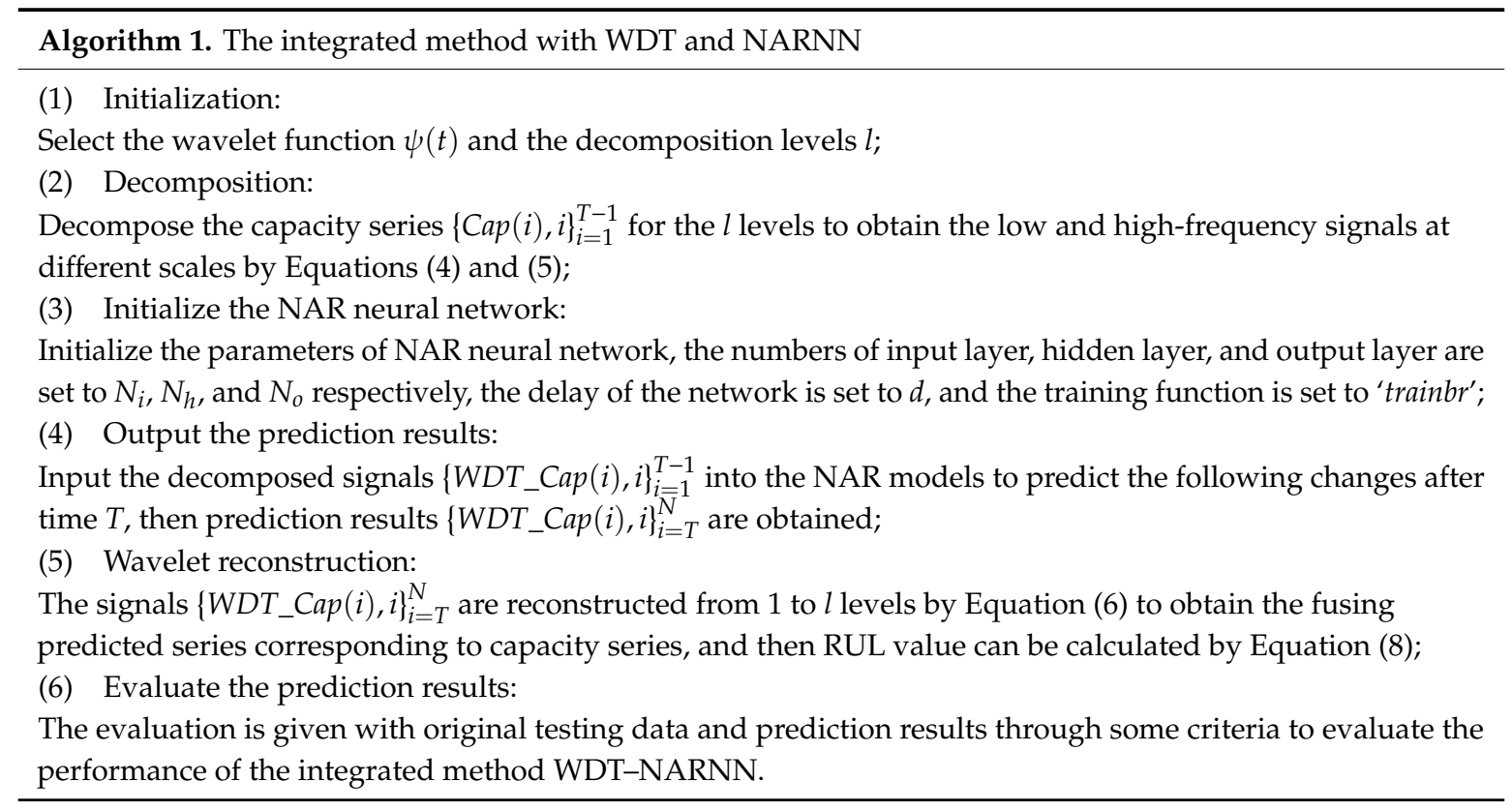

\subsection{Performance Analysis}

We use the following four evaluation criteria to measure and demonstrate the accuracy and stability of the proposed method:

(1) Root Mean Square Error (RMSE) to evaluate the prediction accuracy. The smaller the RMSE is, the better the prediction performance:

$$
R M S E=\sqrt{\frac{\sum_{i=1}^{n}\left(y_{i}-\hat{y}_{i}\right)^{2}}{n}}
$$

(2) $R^{2}$ to evaluate the prediction performance. If the fitting degree between the prediction curve and real curve is high, $R^{2}$ will be close to 1 :

$$
R^{2}(y, \hat{y})=1-\frac{\sum_{i=0}^{n-1}\left(y_{i}-\hat{y}_{i}\right)^{2}}{\sum_{i=0}^{n-1}\left(y_{i}-\bar{y}_{i}\right)^{2}}
$$

(3) Absolute Error $(A E)$ to evaluate the RUL accuracy of the prediction model:

$$
A E=|R-\hat{R}|
$$

(4) Prediction Accuracy Improvement Ratio $\left(\eta_{A E}\right)$ to evaluate the RUL prediction accuracy improvement ratio of two different methods. If $\eta_{A E}>0$, the first method is more accurate, on the contrary, the second method has higher prediction accuracy:

$$
\eta_{A E}=\frac{A E_{2}-A E_{1}}{R}
$$

where $\mathrm{n}$ is the sample size, $y_{i}$ is the real value of battery capacity, $\hat{y}_{i}$ is the predicted value of battery capacity, and $\bar{y}_{i}$ is the mean value of predicted battery capacity. $R$ is the real RUL, $\hat{R}$ is the predicted RUL. 


\section{Results and Discussion}

\subsection{RUL Prediction of Lithium-Ion Battery}

In order to verify the effectiveness of the proposed RUL prediction model for lithium-ion batteries based on WDT-NARNN (named M1), we design two comparative models M2 and M3 as shown in Table 1. Model M2 uses the NAR neural network to directly establish the original capacity time series prediction model without using wavelet decomposition technology to decompose the capacity series. Model M3 uses BP neural network to establish the prediction model, and the other steps are the same as model M1. In this comparison, M2 is tested to analyze the effect of WDT in the proposed model M1. M3 is considered to illustrate the ability of the NAR neural network to predict capacity time series. Experimental setting: We select the data from cycle 1 to cycle 69 as the training samples, and the predicted starting point is 70. The 'dmey' wavelet is selected to carry out 6-layer decomposition of capacity series. The Bayesian regularization algorithm (trainbr) is utilized as the training function of NARNN. The hidden layer nodes are set to 10 and the feedback delay is set to 2 . The training samples of network are divided into $70 \%$ training samples, $15 \%$ verification samples, and $15 \%$ test samples.

Table 1. The proposed three models (M1-M3).

\begin{tabular}{cc}
\hline Model & Model Description \\
\hline M1 & WDT combine with NARNN \\
M2 & NARNN without using WDT \\
M3 & WDT combine with BPNN \\
\hline
\end{tabular}

Figure 5 shows the comparison of prediction results of different models. It can be observed that the prediction curve of model M1 is closest to the real capacity degradation curve and the capturing of capacity regeneration is most accurate. Compared with model M1, the prediction result of model M2 is less effective in capturing capacity regeneration, and is more far away from the real capacity with the increase in cycle numbers. The comparison reflects the validity of using WDT to extract capacity global degradation and local regenerations to build prediction models respectively. Meanwhile, model M3 uses the WDT to separate the global degradation and local regeneration, but the prediction curve of M3 still shows a smooth trend. This phenomenon indicates that the traditional BP neural network does not learn the regeneration phenomenon well in the training stage of network, which is mainly due to its lack of feedback connection structure, resulting in its lack of 'memory' ability. So, BP neural network is not very effective in dealing with RUL prediction of lithium-ion batteries, which is a time series prediction problem. However, the model M1 utilizes NARNN with time series prediction ability that can learn the capacity regeneration well and has a better prediction performance. 


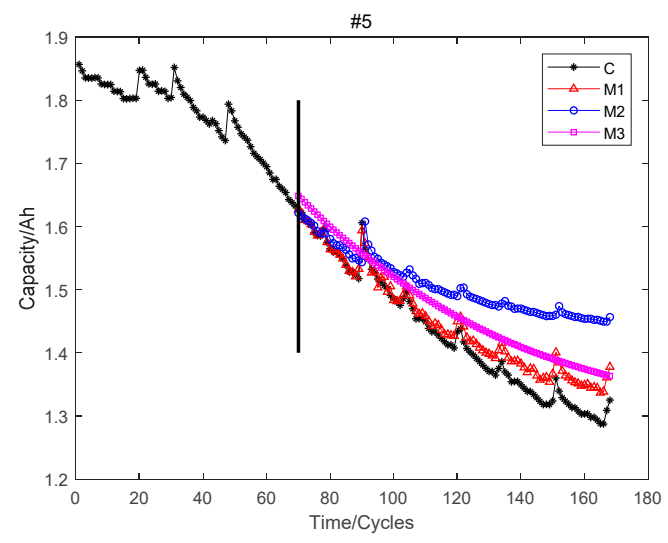

(a)

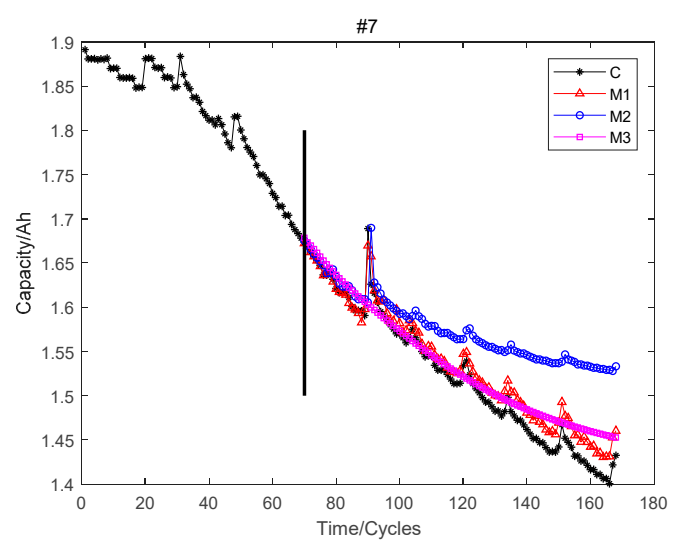

(c)

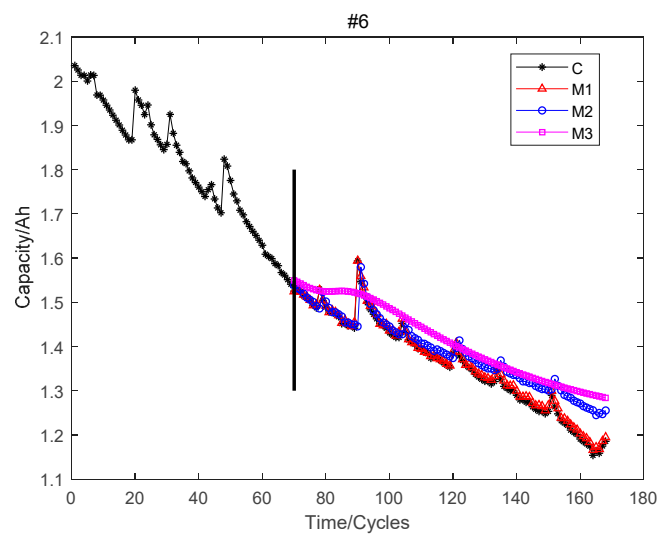

(b)

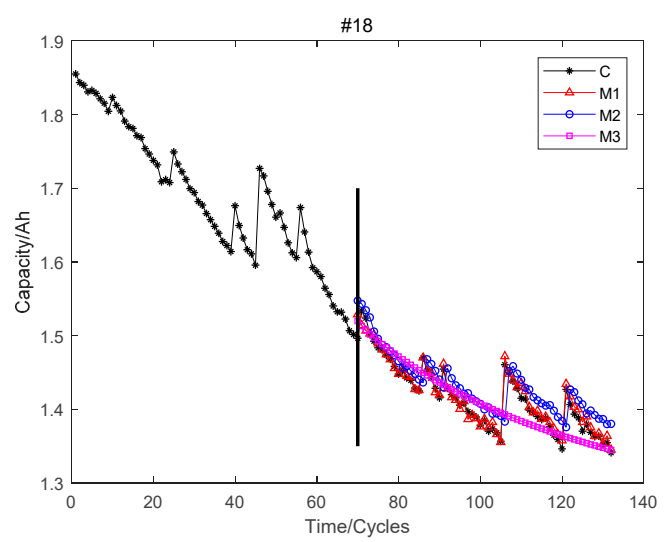

(d)

Figure 5. The comparisons for different models. (a) \#5; (b) \#6; (c) \#7; (d) \#18.

In addition, Table 2 gives the prediction performance of different models for four batteries (\#5, $\# 6$, \#7, and \#18). It can be seen that the RMSE of four batteries predicted by model M1 are all smaller than 0.03 and $\mathrm{R}^{2}$ are all larger than 0.9 , indicating that model M1 has good prediction performance and high fitting degree to the original capacity curve. Meanwhile, as can be seen from columns 3-6 in Table 2, for RMSE, model M1 of each battery is significantly smaller than model M2 and M3. For $\mathrm{R}^{2}, \mathrm{M} 1$ is significantly larger than model M2 and M3. The above analysis illustrates that model M1 can effectively capture the regeneration of capacity and has good prediction performance, and the prediction performance of model M1 is significantly better than that of model M2 and M3.

Table 2. Comparison of different models (M1-M3) for four batteries.

\begin{tabular}{cccccc}
\hline Evaluate Criteria & Model & \#5 & \#6 & \#7 & \#18 \\
\hline \multirow{3}{*}{ RMSE } & M1 & 0.0270 & 0.0087 & 0.0175 & 0.0064 \\
& M2 & 0.0949 & 0.0436 & 0.0678 & 0.0260 \\
& M3 & 0.0500 & 0.0616 & 0.0234 & 0.0253 \\
\hline \multirow{2}{*}{$R^{2}$} & M1 & 0.9226 & 0.9933 & 0.9460 & 0.9751 \\
& M2 & 0.4151 & 0.8457 & 0.4611 & 0.6494 \\
& M3 & 0.7745 & 0.7298 & 0.9035 & 0.6091 \\
\hline
\end{tabular}

\subsection{Different Starting Point Predictions and Comparison}

Considering the EMD decomposition combined with logic regression and Gaussian regression model (named M-LG) proposed in reference [8] also realized the lithium-ion battery health prognostics 
with capturing the capacity regeneration phenomenon. In order to analyze the validity of model M1 more comprehensively, the prediction experiments of model M1 at four different prediction starting points $(60,70,80$, and 90$)$ are designed, and the prediction results of RUL are compared with those of M2, M3, and M-LG models.

Figure 6 shows the capacity series prediction of model M1 at different prediction starting points for four batteries. It can be observed from the overall that, the later the prediction starting point is, the closer the prediction result is to the real value for the four batteries. For battery \#5 and \#7, the effect of prediction starting point on predicted results is relatively obvious. For battery $\# 6$, the predicted curves at the starting points 70,80 , and 90 are near to the real capacity curve. For battery $\# 18$, the prediction results at each starting point are very close to the real value, which means the prediction result is not influenced evidently by the different prediction starting point.

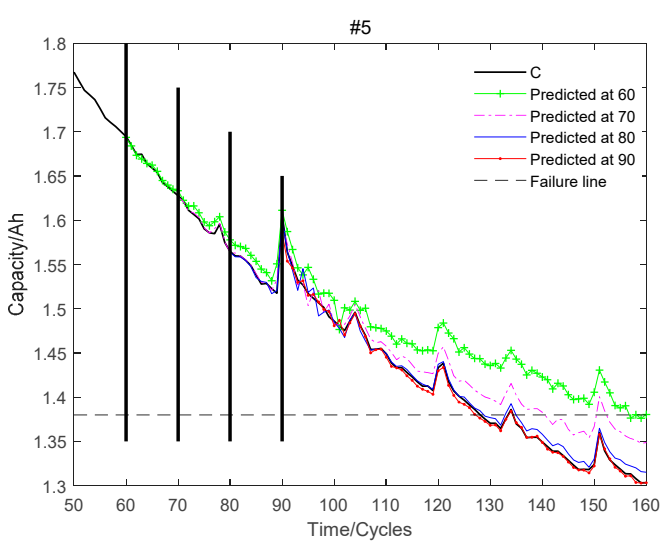

(a)

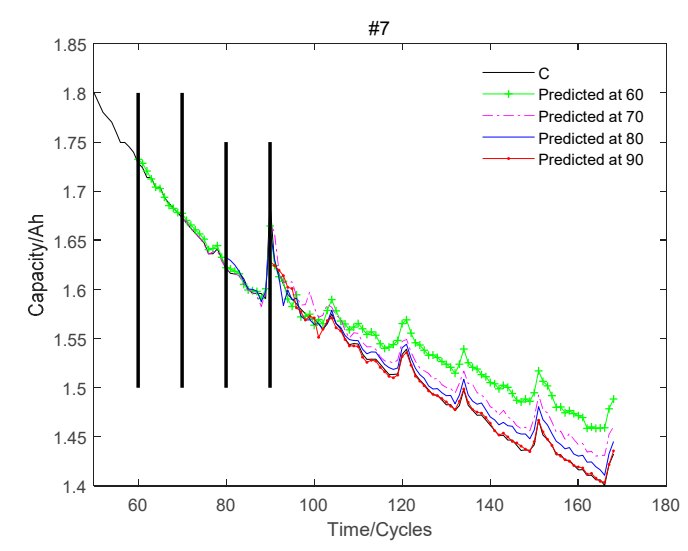

(c)

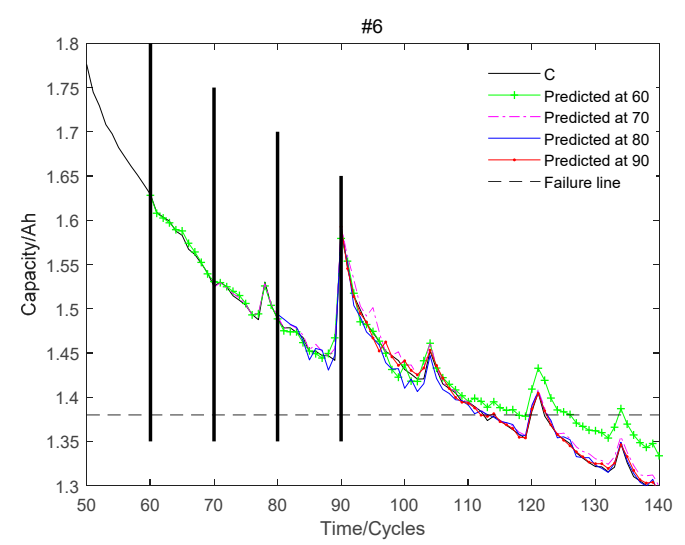

(b)

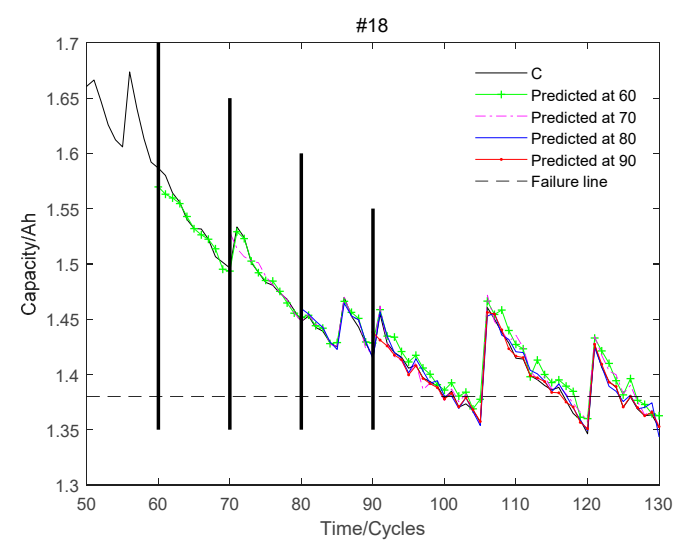

(d)

Figure 6. Capacity series prediction of model M1 at different prediction starting points: (a) battery \#5; (b) battery \#6; (c) battery \#7; (d) battery \#18.

Table 3 gives the RUL prediction results using model M1 at different starting points for battery \#5, \#6, and \#18 (Since the degradation of battery \#7 does not reach the failure threshold, no research is done for the time being). We can see that for each battery, the AE of RUL prediction results decreases with the prediction starting point moving backward. For battery \#5, the RUL AE is equal to 28 and 12 at the prediction starting points 60 and 70, respectively, and the errors are relatively large. However, the RUL AE is 0 and 1 at the prediction starting point 80 and 90 respectively. For battery \#6, the RUL $\mathrm{AE}$ at prediction starting point 60 is 5 , and the RUL AE is less than or equal to 1 at the starting point 70 and later. For battery \#18, the RUL AE at each prediction starting point is less than or equal to 2 , which is obviously less effected by the prediction starting point. The above experimental results 
show that the model M1 is relatively less affected by the prediction starting point, and the prediction performance is relatively stable.

Table 3. RUL prediction results of model M1 at different prediction starting points.

\begin{tabular}{cccc}
\hline Battery & Prediction starting point & Predicted RUL & RUL AE \\
\hline \multirow{3}{*}{$\# 5$} & 60 & 96 & 28 \\
70 & 70 & 12 \\
& 80 & 48 & 0 \\
& 90 & 37 & 1 \\
\hline \multirow{3}{*}{$* 6$} & 60 & 57 & 5 \\
& 70 & 42 & 0 \\
& 80 & 33 & 1 \\
$\# 18$ & 90 & 22 & 0 \\
\hline \multirow{3}{*}{} & 60 & 42 & 2 \\
& 70 & 30 & 0 \\
& 80 & 20 & 0 \\
\hline
\end{tabular}

Besides, we did 50 times prediction experiments for batteries \#5, \#6, and\#18 using model M1 at different prediction starting points respectively, and gave the PDF of predicted End of Life (EOL) in Figure 7 (Color Filling Part), that is, within the PDF distribution range, the battery is likely to reach the EOL. As a whole, each distribution at different starting points is relatively concentrated, and as the predicted starting point moves backward, the center of the PDF is closer to the actual EOL, which indicates that the predicted result is more accurate.

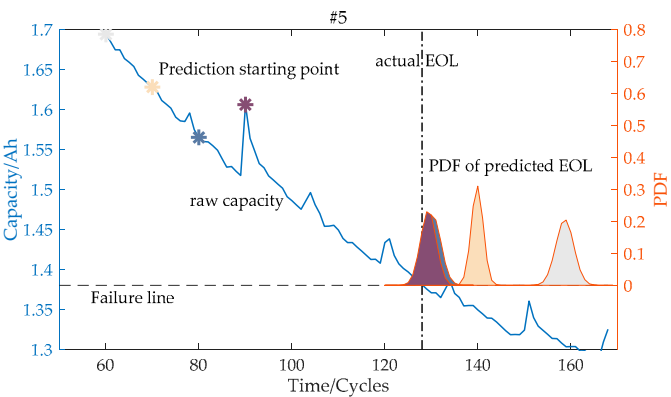

(a)

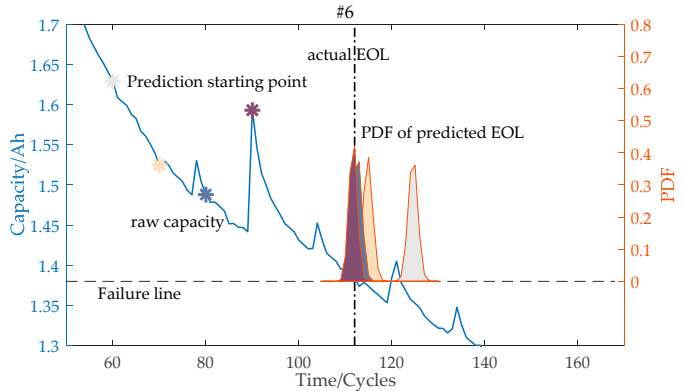

(b)

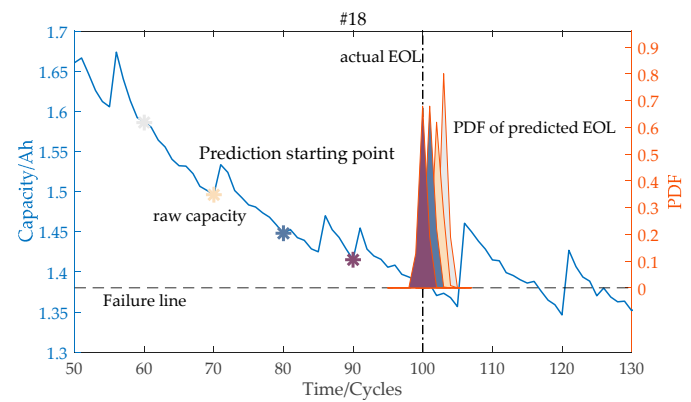

(c)

Figure 7. Uncertainty of predicted End of Life (EOL) at different prediction starting points. (a) battery \#5; (b) battery \#6; (c) battery \#18.

Table 4 gives the comparison of average RUL AE and average $\eta_{A E}$ under four different starting points of models M1, M2, M3, and M-LG for three batteries, where $\eta_{A E}$ is the prediction accuracy improvement ratio of model M1 corresponding to other models, so the average $\eta_{A E}$ of M1 is null. As 
can be seen from the third column, for batteries \#5, \#6, and \#18, the average RUL AE of model M1 is obviously smaller than that of other models. From the fourth column, we can see that the average $\eta_{A E}$ is between $14.3 \%$ and $54.9 \%$, which shows the prediction performance of model M1 is obviously better than that of model M2, M3, and M-LG on the whole. All the above experiments show that the proposed WDT-NARNN method is reasonable and suitable for RUL prediction of lithium-ion batteries with capturing capacity regeneration phenomenon.

Table 4. Comparisons of the average RUL prediction accuracy at four prediction starting points under model M1 and other three methods (M2, M3, and M-LG).

\begin{tabular}{cccc}
\hline Battery & Method & Average RUL AE & Average $\eta_{A E}$ \\
\hline \multirow{3}{*}{$\# 5$} & M1 & 10.3 & - \\
& M2 & 14 & $34.2 \%$ \\
& M3 & 12 & $21.1 \%$ \\
M-LG & 16.3 & $14.3 \%$ \\
\hline \multirow{2}{*}{$* 6$} & M1 & 1.5 & - \\
& M2 & 17.8 & $37.8 \%$ \\
& M3 & 12 & $34.6 \%$ \\
M-LG & 22.3 & $54.9 \%$ \\
\hline \multirow{3}{*}{$\# 18$} & M1 & 0.5 & - \\
& M2 & 6.5 & $35.6 \%$ \\
& M3 & 14.8 & $50 \%$ \\
& M-LG & 6 & $25.8 \%$ \\
\hline
\end{tabular}

\section{Conclusions}

Considering that the capacity regeneration phenomenon has a great impact on the RUL prediction of lithium-ion batteries, in order to improve the RUL prediction accuracy, a WDT-NARNN method with considering capacity regeneration phenomenon is proposed. Firstly, the Wavelet decomposition technology is used to decompose the capacity time series at multi-scales to extract the global degradation part and local regeneration part. Then, the time series prediction models based on NAR neural network for the two parts are constructed respectively. Finally, the two parts of the prediction results are combined to obtain the final RUL prediction value. Experimental results show that the proposed method can effectively capture the regeneration phenomenon and has high prediction accuracy. Additionally, the prediction performance is stable and less affected by different prediction starting point.

Author Contributions: Conceptualization, X.P.; methodology, X.P.; validation, R.H.; writing—original draft preparation, R.H.; writing—review and editing, X.P.; visualization, R.H.; supervision, J.W., Y.S., J.J. and J.Z.

Funding: This work was partially supported by National Natural Science Foundation of China under Grant 61533013, the key Program of Research and Development of ShanXi Province under Grand 201703D111011, and the Natural Science Foundation of ShanXi Province under Grant 201801D121159, 201801D221208.

Conflicts of Interest: The authors declare no conflict of interest.

\section{References}

1. Liu, D.T.; Zhou, J.B.; Guo, L.M.; Peng, Y. Survey on lithium-ion battery health assessment and cycle life estimation. Chin. J. Sci. Instrum. 2015, 36, 1-16. [CrossRef]

2. Lukic, S.M.; Cao, J.; Bansal, R.C.; Rodriguez, F.; Emadi, A. Energy storage systems for automotive applications. IEEE Trans. Ind. Electron. 2008, 55, 2258-2267. [CrossRef]

3. Nishi, Y. Lithium-ion secondary batteries. Power Sour. 2001, 100, 101-106. [CrossRef]

4. Saha, B.; Goebel, K.; Poll, S.; Christophersen, J. Prognostics Methods for Battery Health Monitoring Using a Bayesian Framework. IEEE Trans. Instrum. Meas. 2009, 58, 291-296. [CrossRef]

5. Goebel, K.; Saha, B.; Saxena, A.; Celaya, J.R.; Christophersen, J.P. Prognostics in battery health management. IEEE Instrum. Meas. Mag. 2008, 11, 33-40. [CrossRef] 
6. Williard, N.; He, W.; Hendricks, C.; Pecht, M. Lessons Learned from the 787 Dreamliner Issue on Lithium-Ion Battery Reliability. Energies 2013, 6, 4682-4695. [CrossRef]

7. Lu, L.G.; Han, X.B.; Li, J.Q.; Hua, J.F.; Ouyang, M.G. A review on the key issues for lithium-ion battery management in electric vehicles. J. Power Sour. 2013, 226, 272-288. [CrossRef]

8. Yu, J.B. State of health prediction of lithium-ion batteries: Multiscale logic regression and Gaussian process regression ensemble. Reliab. Eng. Syst. Saf. 2018, 174, 82-95. [CrossRef]

9. Sun, Y.H.; Jou, H.L.; Wu, J.C. Aging Estimation Method for Lead-Acid Battery. IEEE Trans. Energy Convers. 2011, 26, 264-271. [CrossRef]

10. Yu, J.B. State-of-Health Monitoring and Prediction of Lithium-Ion Battery Using Probabilistic Indication and State-Space Model. IEEE Trans. Instrum. Meas. 2015, 64, 2937-2949. [CrossRef]

11. Liu, D.T.; Pang, J.Y.; Zhou, J.B.; Peng, Y.; Pecht, M. Prognostics for state of health estimation of lithium-ion batteries based on combination Gaussian process functional regression. Microelectron. Reliab. 2013, 53, 832-839. [CrossRef]

12. Saha, B.; Goebel, K. Modeling Li-ion Battery Capacity Depletion in a Particle Filtering Framework. In Proceedings of the Annual Conference of the Prognostics and Health Management Society, San Diego, CA, USA, 27 September-1 October 2009.

13. He, Y.J.; Shen, J.N.; Shen, J.F.; Ma, Z.F. State of health estimation of lithium-ion batteries: A multiscale Gaussian process regression modeling approach. Aiche J. 2015, 61, 1589-1600. [CrossRef]

14. Song, Y.C.; Liu, D.T.; Yang, C.; Peng, Y. Data-driven hybrid remaining useful life estimation approach for spacecraft lithium-ion battery. Microelectron. Reliab. 2017, 75, 142-153. [CrossRef]

15. Gao, D.; Huang, M.H. Prediction of Remaining Useful Life of Lithium-ion Battery based on Multi-kernel Support Vector Machine with Particle Swarm Optimization. J. Power Electron. 2017, 17, 1288-1297. [CrossRef]

16. Qin, T.C.; Zeng, S.K.; Guo, J.B. Robust prognostics for state of health estimation of lithium-ion batteries based on an improved PSO-SVR model. Microelectron. Reliab. 2015, 55, 1280-1284. [CrossRef]

17. Song, Y.C.; Liu, D.T.; Hou, Y.D.; Yu, J.X.; Peng, Y. Satellite lithium-ion battery remaining useful life estimation with an iterative updated RVM fused with the KF algorithm. Chin. J. Aeronaut. 2018, 31, 31-40. [CrossRef]

18. Wu, J.; Zhang, C.B.; Chen, Z.H. An online method for lithium-ion battery remaining useful life estimation using importance sampling and neural networks. Appl. Energy 2016, 173, 134-140. [CrossRef]

19. Liu, D.T.; Yin, X.H.; Song, Y.C.; Liu, W.; Peng, Y. An On-Line State of Health Estimation of Lithium-Ion Battery Using Unscented Particle Filter. IEEE Access 2018, 6, 40990-41001. [CrossRef]

20. Peng, Y.; Hou, Y.D.; Song, Y.C.; Pang, J.Y.; Liu, D.T. Lithium-Ion Battery Prognostics with Hybrid Gaussian Process Function Regression. Energies 2018, 11, 1420. [CrossRef]

21. Deng, L.M.; Hsu, Y.C.; Li, H.X. An Improved Model for Remaining Useful Life Prediction on Capacity Degradation and Regeneration of Lithium-Ion Battery. In Proceedings of the Annual Conference of the Prognostics and Health Management Society, Saint Petersburg, FL, USA, 2-7 October 2017.

22. Liu, D.T.; Zhou, J.B.; Liao, H.T.; Peng, Y.; Peng, X.Y. A Health Indicator Extraction and Optimization Framework for Lithium-Ion Battery Degradation Modeling and Prognostics. IEEE Trans. Syst. Man Cybern. Syst. 2015, 45, 915-928. [CrossRef]

23. Zangenehmadar, Z.; Moselhi, O. Assessment of Remaining Useful Life of Pipelines Using Different Artificial Neural Networks Models. J. Perform. Constr. Facil. 2016, 30. [CrossRef]

24. Zhang, X.H.; Xiao, L.; Kang, J.S. Degradation Prediction Model Based on a Neural Network with Dynamic Windows. Sensors 2015, 15, 6996-7015. [CrossRef] [PubMed]

25. Li, M.B.; Ji, S.W.; Liu, G. Forecasting of Chinese E-Commerce Sales: An Empirical Comparison of ARIMA, Nonlinear Autoregressive Neural Network, and a Combined ARIMA-NARNN Model. Math. Probl. Eng. 2018, 2018, 1-13. [CrossRef]

26. Wang, K.W.; Deng, C.; Li, J.P.; Zhang, Y.Y.; Li, X.Y.; Wu, M.C. Hybrid methodology for tuberculosis incidence time-series forecasting based on ARIMA and a NAR neural network. Epidemiol. Infect. 2017, 145, 1118-1129. [CrossRef]

27. Zhang, Y.Z.; Xiong, R.; He, H.W.; Pecht, M.G. Long Short-Term Memory Recurrent Neural Network for Remaining Useful Life Prediction of Lithium-Ion Batteries. IEEE Trans. Veh. Technol. 2018, 67, 5695-5705. [CrossRef]

28. Du, Z.H.; Qin, M.J.; Zhang, F.; Liu, R.Y. Multistep-ahead forecasting of chlorophyll a using a wavelet nonlinear autoregressive network. Knowl. Based Syst. 2018, 160, 61-70. [CrossRef] 
29. Ibrahim, M.; Jemei, S.; Wimmer, G.; Hissel, D. Nonlinear autoregressive neural network in an energy management strategy for battery/ultra-capacitor hybrid electrical vehicles. Electr. Power Syst. Res. 2016, 136, 262-269. [CrossRef]

30. Benmouiza, K.; Cheknane, A. Small-scale solar radiation forecasting using ARMA and nonlinear autoregressive neural network models. Theor. Appl. Climatol. 2016, 124, 945-958. [CrossRef]

31. Rai, A.; Upadhyay, S.H. The use of MD-CUMSUM and NARX neural network for anticipating the remaining useful life of bearings. Measurement 2017, 111, 397-410. [CrossRef]

32. Gilles, J. Empirical Wavelet Transform. IEEE Trans. Signal Process. 2013, 61, 3999-4010. [CrossRef]

33. Bhattacharyya, A.; Singh, L.; Pachori, R.B. Fourier-Bessel series expansion based empirical wavelet transform for analysis of non-stationary signals. Digit Signal Process. 2018, 78, 185-196. [CrossRef]

34. Amezquita-Sanchez, J.P.; Adeli, H. A new music-empirical wavelet transform methodology for time-frequency analysis of noisy nonlinear and non-stationary signals. Digit. Signal Process. 2015, 45, 55-68. [CrossRef]

35. Tobon-Mejia, D.A.; Medjaher, K.; Zerhouni, N.; Tripot, G. Estimation of the Remaining Useful Life by Using Wavelet Packet Decomposition and HMMs. In Proceedings of the IEEE Aerospace Conference, Big Sky, MT, USA, 5-12 March 2011.

36. Saha, B.; Goebel, K. Battery Data Set. Available online: https:/ti.arc.nasa.gov/tech/dash/groups/pcoe/ prognostic-data-repository/ (accessed on 20 October 2018).

(C) 2019 by the authors. Licensee MDPI, Basel, Switzerland. This article is an open access article distributed under the terms and conditions of the Creative Commons Attribution (CC BY) license (http://creativecommons.org/licenses/by/4.0/). 